Disclosure of Interest: None declared

DOI: 10.1136/annrheumdis-2017-eular.4252

\section{SAT0179 THE ASP358ALA VARIANT IN THE IL6R GENE IS SIGNIFICANTLY ASSOCIATED WITH DIFFERENCES IN SOLUBLE IL-6R PROTEIN LEVELS BUT NOT WITH DIFFERENCES IN SARILUMAB RESPONSE IN RHEUMATOID ARTHRITIS (RA) PATIENTS}

A. Damask ${ }^{1}$, A. Boyapati ${ }^{1}$, J.D. Hamilton ${ }^{1}$, S. Hamon ${ }^{1}$, C. Paccard ${ }^{2}$, J. Parrino ${ }^{1}$, J. van Adelsberg ${ }^{1}$, N.M. Graham ${ }^{1}$, J. Penn ${ }^{1}$, A. Lopez ${ }^{1}$, J. Reid ${ }^{1}$, J. Overton ${ }^{1}$, A. Baras ${ }^{1}$, A.R. Shuldiner ${ }^{1}$, C. Paulding ${ }^{1} .{ }^{1}$ Regeneron Pharmaceuticals, Inc, Tarrytown, United States; ${ }^{2}$ Sanofi R\&D, Chilly-Mazarin, France

Background: Sarilumab is a human mAb that blocks IL-6 from binding to both membrane-bound and soluble IL-6R $\alpha$ (sIL-6R). A missense variant in the IL6R gene, Asp358Ala (rs2228145), falls within a proteolytic cleavage site and individuals with an alanine at this position have increased SIL-6R in circulation. ${ }^{1}$ In addition, this variant has been associated with several diseases including RA. ${ }^{2}$ Objectives: To determine the impact of the Asp358Ala variant on SIL-6R concentrations and response of RA patients to sarilumab.

Methods: DNA was collected from patients enrolled in the MOBILITY study (NCT01061736) that evaluated the efficacy and safety of sarilumab + methotrexate (MTX) in RA patients with inadequate response to MTX. The pharmacogenetic analysis was conducted on 599 Caucasian patients (396 sarilumab 150 or 200 mg q2w + MTX, 203 placebo + MTX)

Results: Concentrations of sIL-6R were strongly associated with the Asp358Ala genotypes at baseline $\left(p=4.2 \times 10^{-11}\right)$. The difference in sIL-6R concentrations between genotype groups continued to increase in sarilumab-treated patients through the end of treatment, particularly for the CC genotype (Figure; $p=7.8$ $\times 10^{-12}$ ). There was a modest association for change in sIL-6R in placebo + MTX-treated patients $(p=0.0052)$. Variation in Asp358Ala was not associated with sarilumab efficacy, including mTSS at week 52 and ACR scores (Table).

Table 1. Efficacy Endpoints ${ }^{1}$ and sIL-6R Levels by Asp358Ala Genotypes in Sarilumab-Treated Patients ${ }^{2}$

\begin{tabular}{lcccccccc}
\hline Genotype & slL-6R & mTSS $^{3}$ & ACR20 & ACR50 & ACR70 & MCR $^{4}$ & SJC $^{5}$ & TJC $^{6}$ \\
\hline AA $(n=129)$ & $317.7(21.0)$ & $1.4(0.5)$ & 59 & 40 & 22 & 13 & $-10.9(0.8)$ & $-16.5(1.2)$ \\
AC $(n=190)$ & $362.3(16.4)$ & $0.7(0.3)$ & 65 & 43 & 22 & 12 & $-11.1(0.6)$ & $-18.3(1.0)$ \\
CC $(n=77)$ & $470.8(22.8)$ & $0.9(0.5)$ & 75 & 48 & 31 & 17 & $-11.6(1.0)$ & $-19.1(1.5)$
\end{tabular}

\begin{tabular}{lllllllll}
$p$ value & $7.8 \times 10^{-12}$ & 0.89 & 0.06 & 0.67 & 0.57 & 0.71 & 0.25 & 0.20 \\
\hline
\end{tabular}

${ }^{1}$ sIL-6R, mTSS, SJC, TJC show LS mean (SE). Wk 24 ACR20, ACR50, ACR70, and MCR show $\%$ achieving endpoint. ${ }^{2} 150$ or $200 \mathrm{mg}$ q2w doses combined. ${ }^{3}$ van der Heijde modified total Sharp score at wk 52. ${ }^{4}$ Major clinical response is defined as achieving and maintaining ACR70 for $\geq 24$ consecutive weeks during the 52 -wk period. ${ }^{5}$ Swollen joint count. ${ }^{6}$ Tender joint count.
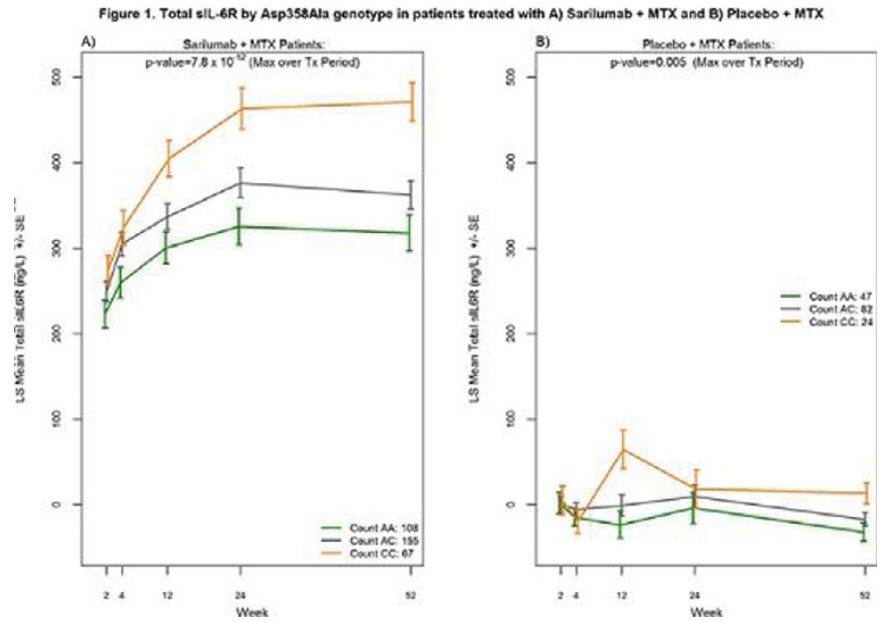

Conclusions: The Asp358Ala variant in the IL6R gene is significantly associated with differences in sIL-6R levels at baseline and after sarilumab treatment. The differences across genotypes may be due to increases in sIL-6R production. Importantly, this variant was not associated with differences in sarilumab treatment response. These data suggest that the sarilumab doses used for this clinical study saturate both the membrane and soluble forms of IL-6R and effectively block IL-6 signaling. Sarilumab provides therapeutic benefit for RA patients irrespective of their Asp358Ala genotype status.

References:

[1] Garbers et al. Biochim Biophys Acta. 2014;1842:1485-1494.

[2] Okada et al. Nature. 2014;506:376-381.

Acknowledgements: This study was sponsored by Sanofi Genzyme and Regeneron Pharmaceuticals, Inc. Editorial support was provided by MedThink SciCom and funded by Sanofi Genzyme and Regeneron Pharmaceuticals, Inc

Disclosure of Interest: A. Damask Shareholder of: Regeneron Pharmaceuticals, Inc, Employee of: Regeneron Pharmaceuticals, Inc, A. Boyapati Shareholder of: Regeneron Pharmaceuticals, Inc, Employee of: Regeneron Pharmaceuticals, Inc, J. Hamilton Shareholder of: Regeneron Pharmaceuticals, Inc, Employee of: Regeneron Pharmaceuticals, Inc, S. Hamon Shareholder of: Regeneron Pharmaceuticals, Inc, Employee of: Regeneron Pharmaceuticals, Inc, C. Paccard Shareholder of: Sanofi R\&D, Employee of: Sanofi R\&D, J. Parrino Shareholder of: Regeneron Pharmaceuticals, Inc, Employee of: Regeneron Pharmaceuticals, Inc, J. van Adelsberg Shareholder of: Regeneron Pharmaceuticals, Inc, Employee of: Regeneron Pharmaceuticals, Inc, N. Graham Shareholder of: Regeneron Pharmaceuticals, Inc, Employee of: Regeneron Pharmaceuticals, Inc, J. Penn Shareholder of: Regeneron Pharmaceuticals, Inc, Employee of: Regeneron Pharmaceuticals, Inc, A. Lopez Shareholder of: Regeneron Pharmaceuticals, Inc, Employee of: Regeneron Pharmaceuticals, Inc, J. Reid Shareholder of: Regeneron Pharmaceuticals, Inc, Employee of: Regeneron Pharmaceuticals, Inc, J. Overton Shareholder of: Regeneron Pharmaceuticals, Inc, Employee of: Regeneron Pharmaceuticals, Inc, A. Baras Shareholder of: Regeneron Pharmaceuticals, Inc, Employee of: Regeneron Pharmaceuticals, Inc, A. Shuldiner Shareholder of: Regeneron Pharmaceuticals, Inc, Employee of: Regeneron Pharmaceuticals, Inc, C. Paulding Shareholder of: Regeneron Pharmaceuticals, Inc, Employee of: Regeneron Pharmaceuticals, Inc

DOI: 10.1136/annrheumdis-2017-eular.4983

\section{SAT0180 EFFICACY AND SAFETY OF SARILUMAB 200 MG Q2W ADMINISTERED AS COMBINATION THERAPY OR MONOTHERAPY IN DIFFERENT PATIENT POPULATIONS WITH} ACTIVE RA

M.C. Genovese ${ }^{1}$, R. Fleischmann ${ }^{2}$, H. van Hoogstraten ${ }^{3}$, E.K. Mangan ${ }^{4}$, S. Jayawardena ${ }^{3}$, G.R. Burmester ${ }^{5}$. ${ }^{1}$ Stanford University Medical Center, Palo Alto; ${ }^{2}$ Metroplex Clinical Research Center, University of Texas Southwestern Medical Center, Dallas; ${ }^{3}$ Sanofi Genzyme, Bridgewater $;{ }^{4}$ Regeneron Pharmaceuticals, Inc, Tarrytown, United States; ${ }^{5}$ Charité - University Medicine Berlin, Free University and Humboldt University of Berlin, Berlin, Germany

Background: Sarilumab, a human mAb blocking the IL-6R $\alpha$, was evaluated in 3 pivotal clinical trials.

Objectives: To assess efficacy and safety of sarilumab $200 \mathrm{mg}$ q2w + csDMARDs or as monotherapy (MONARCH) in adults with active RA and inadequate response or intolerance to MTX (MOBILITY/MONARCH) or TNFi (TARGET)

Methods: MOBILITY (NCT01061736) was a 52-wk study; TARGET (NCT01709578) and MONARCH (NCT02332590) were 24-wk studies. Patients were randomized to placebo (Pbo) or SC sarilumab 150 or $200 \mathrm{mg} \mathrm{q2w}+$ MTX (MOBILITY) or csDMARDs (TARGET). MONARCH patients were randomized to SC monotherapy with adalimumab $40 \mathrm{mg}$ q2w or sarilumab $200 \mathrm{mg}$ q2w. Efficacy endpoints assessed in all 3 studies will be presented.

Results: Within studies, baseline demographic and disease characteristics were similar among treatment groups. Sarilumab $200 \mathrm{mg}$ q2w improved ACR responses, HAQ-DI, DAS28-CRP, and CDAI (Table). Treatment response with sarilumab + cSDMARDs was similar in MTX-IR and TNF-IR patients and with sarilumab monotherapy. Incidence of TEAEs and SAEs with sarilumab was more frequent vs Pbo (MOBILITY, TARGET) and similar to adalimumab (MONARCH). The most common TEAEs included infections, neutropenia, and injection site reactions and occurred more often with sarilumab vs Pbo (MOBILITY, TARGET). In MONARCH, rates of infection were similar with sarilumab and adalimumab,

Abstract SAT0180 - Table 1. Sarilumab Efficacy in 3 Phase 3 Studies

\begin{tabular}{|c|c|c|c|c|c|c|}
\hline & \multicolumn{2}{|r|}{ MOBILITY } & \multicolumn{2}{|r|}{ TARGET } & \multicolumn{2}{|c|}{ MONARCH } \\
\hline & $\begin{array}{l}\mathrm{Pbo}+\mathrm{MTX} \\
(\mathrm{N}=398)\end{array}$ & $\begin{array}{c}\text { Sarilumab } 00 \mathrm{mg} \text { q2w + MTX } \\
\qquad(\mathrm{N}=399)\end{array}$ & $\begin{array}{c}\text { Pbo + csDMARDs } \\
(\mathrm{N}=181)\end{array}$ & $\begin{array}{c}\text { Sarilumab } 200 \text { mg q2w + csDMARDs } \\
(\mathrm{N}=184)\end{array}$ & $\begin{array}{l}\text { Adalimumab 40mg q2w } \\
(\mathrm{N}=185)\end{array}$ & $\begin{array}{c}\text { Sarilumab } 200 \mathrm{mg} \mathrm{q2w} \\
(\mathrm{N}=184)\end{array}$ \\
\hline ACR20, wk 24, \% & 33.4 & $66.4^{\star}$ & 33.7 & $60.9^{*}$ & 58.4 & $71.7^{\dagger}$ \\
\hline ACR50, wk 24, \% & 16.6 & $45.6^{*}$ & 18.2 & $40.8^{*}$ & 29.7 & $45.7^{\dagger}$ \\
\hline ACR70, wk 24, \% & 7.3 & $24.8^{*}$ & 7.2 & $16.3^{*}$ & 11.9 & $23.4^{\dagger}$ \\
\hline \multicolumn{7}{|c|}{$\mathrm{HAQ}-\mathrm{DI}$, mean change from baseline $\pm \mathrm{SD}$} \\
\hline Wk 16/12/24 & $-0.3 \pm 0.6$ & $-0.6 \pm 0.6^{*}$ & $-0.3 \pm 0.5$ & $-0.5 \pm 0.6^{\star}$ & $-0.4 \pm 0.6$ & $-0.6 \pm 0.7^{\dagger}$ \\
\hline \multicolumn{7}{|c|}{ DAS28-CRP, mean change from baseline $\pm S D$} \\
\hline Wk 24 & $-1.6 \pm 1.4$ & $-3.0 \pm 1.3^{*}$ & $-2.0 \pm 1.2$ & $-3.2 \pm 1.3^{\star}$ & $-2.1 \pm 1.2$ & $-2.9 \pm 1.3^{\ddagger}$ \\
\hline \multicolumn{7}{|c|}{$\mathrm{CDAl}$, mean change from baseline $\pm \mathrm{SD}$} \\
\hline Wk 24 & $-20.3 \pm 15.8$ & $-27.9 \pm 13.2^{*}$ & $-23.9 \pm 12.9$ & $-30.4 \pm 14.5^{\star}$ & $-25.5 \pm 12.9$ & $-29.7 \pm 12.7^{\ddagger}$ \\
\hline
\end{tabular}

${ }^{*} P<0.01$ vs Pbo. ${ }^{\dagger} P<0.01$ vs adalimumab. ${ }^{\ddagger}$ Nominal $P<0.01$ vs adalimumab. 
although neutropenia was more frequent with sarilumab. Safety of sarilumab was generally comparable in monotherapy and combination studies; monotherapy was associated with fewer ALT elevations $>3 \times$ ULN compared with combination therapy: MONARCH, 3\%; MOBILITY, $8 \%$; TARGET, $4 \%$.

Conclusions: Sarilumab $200 \mathrm{mg}$ q2w + csDMARDs significantly reduced disease activity and improved physical function to a similar extent regardless of population (MTX-IR or TNF-IR) and as monotherapy. Safety profile of sarilumab was generally comparable across all 3 trials, with monotherapy resulting in fewer ALT elevations. Acknowledgements: This study was sponsored by Sanofi Genzyme and Regeneron Pharmaceuticals, Inc. Editorial assistance was provided by MedThink SciCom and funded by Sanofi Genzyme and Regeneron Pharmaceuticals, Inc. Disclosure of Interest: M. Genovese Grant/research support from: Roche, Sanofi, GlaxoSmithKline, R-Pharma, RuiYi, and Bristol-Myers Squibb, Consultant for: Roche, Sanofi, GlaxoSmithKline, R-Pharma, RuiYi, and Bristol-Myers Squibb, R. Fleischmann Grant/research support from: AbbVie, Amgen, Ardea, AstraZeneca, Bristol-Myers Squibb, Celgene, GlaxoSmithKline, Janssen, Eli Lilly, Merck, Pfizer, Roche, Sanofi, and UCB, Consultant for: AbbVie, Akros, Amgen, AstraZeneca, Bristol-Myers Squibb, Janssen, Eli Lilly, Pfizer, Roche, and UCB, H. van Hoogstraten Shareholder of: Sanofi Genzyme, Employee of: Sanofi Genzyme, E. Mangan Shareholder of: Regeneron Pharmaceuticals, Inc, Employee of: Regeneron Pharmaceuticals, Inc, S. Jayawardena Shareholder of: Sanofi Genzyme, Employee of: Sanofi Genzyme, G. Burmester Grant/research support from: AbbVie, Bristol-Myers Squibb, Medlmmune, Merck, Pfizer, Roche, and UCB, Consultant for: AbbVie, Bristol-Myers Squibb, Medlmmune, Merck, Pfizer, Roche, and UCB, Speakers bureau: AbbVie, Bristol-Myers Squibb, Merck, Pfizer, Roche, and $U C B$

DOI: 10.1136/annrheumdis-2017-eular.1275

\section{SAT0181 LOW DOSE INTERLEUKIN-2 COMBINED WITH TOCILIZUMAB SELECTIVELY INCREASES REGULATORY T CELLS HELPING REFRACTORY RHEUMATOID ARTHRITIS PATIENTS ACHIEVE REMISSION MORE RAPIDLY}

Z. Sheng-Xiao ${ }^{1}$, M. Xiao-Wen ${ }^{2}$, L. Xiao-Qing ${ }^{1}$, M. Miao ${ }^{1}$, W. Xiao-Yan ${ }^{1}$, N. Hong-Qing ${ }^{2}$, W. Cai-Hong ${ }^{2}$, L. Xiao-Feng ${ }^{1} .{ }^{1}$ Rheumatology; ${ }^{2}$ The Second Hospital of Shanxi Medical University, Taiyuan, China

Background: Rheumatoid arthritis (RA) is a prevalent chronic autoimmune inflammatory disease. Its pathogenesis is closely associated with a failure of endogenous immune tolerance that caused by the imbalance of pro-inflammatory T helper 17 (Th17) cells and anti-inflammatory regulatory T (Treg) cells. Lowdose Interleukin-2 (IL-2) has been showed to induce both Th17 and Treg cells' expansion and activation while IL-6 antagonist Tocilizumab suppresses the differentiation of Th17, which is expected to control the development of RA.

Objectives: To study the influence of the combination of IL-2 and Tocilizumab on $T$ cells subgroups and its clinical efficacy and safety on refractory RA.

Methods: Total 50 RA patients with low Treg cells, who had been treated with glucocorticoids and DMARDs for over 6 months, were divided into three groups randomly. Patients in non-IL-2 group $(n=15)$ were still given conventional glucocorticoids and DMARDs. Patients in IL-2 group $(n=26)$ were not only given those treatments, but injected subcutaneously human IL-2 (aldesleukin) at 50 WIU per day for a 5 day course. Patients in IL-2 and Tocilizumab group $(n=9)$ were not only received the treatment like IL-2 group, but also treated with Tocilizumab at the dosage of $160 \mathrm{mg}$ during the day 1 and day 3 . The demographic features, clinical manifestations and laboratory indicators were compared before and after the treatment.

Results: There was no difference among all groups in gender, age and course of the disease $(p>0.05)$. The ratios of Th1/Th2 and Th17/Treg were significant correlated with ESR, the number of tender or swollen joints and DAS28-ESR $(p<0.05)$ in all three groups of patients. After treatment, the number of Th17 cells and Treg cells was significantly increased in IL-2 group $(p<0.01)$. In IL-2/Tocilizumab group after the treatment, the number of Treg cells were also significantly increased $(p<0.05)$, but not the Th17 cells $(p>0.05)$, leading to a quickly decrease in their ratio $(p<0.05)$. Before the treatment, there was no difference in clinical manifestations among all three groups $(p>0.05)$, but compared with non-IL-2 group, there was a significantly decrease in the number of tender joints $(p<0.01)$ or swollen joints $(p<0.05)$ and DAS28-ESR $(p<0.01)$ in IL-2 group and IL-2/Tocilizumab group after the treatment. Patients in IL-2/ Tocilizumab group had better clinical manifestations' remission although no significant difference compared with IL-2 group $(p>0.05)$. There was no difference in blood routine, liver and renal functions both before and after the treatment among all groups $(p>0.05)$.

Conclusions: IL-2 can effectively increase the level of Treg cells as well as that of Th17 to some degree; while IL-2 combined with Tocilizumab only effectively expand Treg cell number without Th17 increasing, thereby quickly recovers the balance of Th17 and Treg cells. This combination selectively stimulate Treg Cells leading to induce autoimmune tolerance, and seems to help RA patients achieve remission in a rapid way without over-treatment and evaluated side effect, though the long term benefits of this therapy are required to further study in more patients. Acknowledgements: The authors thank Chong Gao for the assistance.

Disclosure of Interest: None declared

DOI: 10.1136/annrheumdis-2017-eular.3611

\section{SAT0182 SIRUKUMAB LEADS TO SIGNIFICANT AND CLINICALLY MEANINGFUL IMPROVEMENTS IN HEALTH-RELATED QUALITY OF LIFE THAT MEET OR EXCEED NORMATIVE VALUES IN PATIENTS WITH RHEUMATOID ARTHRITIS REFRACTORY TO TNF INHIBITORS IN POST HOC ANALYSES OF A PHASE 3 TRIAL}

V. Strand ${ }^{1}$, R. Ganguly ${ }^{2}, \mathrm{~N} . \mathrm{Li}^{3}, \mathrm{~K}$. McQuarrie ${ }^{3} .{ }^{1}$ Stanford University, Palo Alto, $C A ;{ }^{2}$ GlaxoSmithKline, Collegeville, PA; ${ }^{3}$ Janssen Research \& Development, LLC, Spring House, PA, United States

Background: Patients (pts) with rheumatoid arthritis (RA) experience reduced health-related quality of life (HRQoL). Sirukumab (SIR) is an anti-interleukin-6 (IL-6) monoclonal antibody.

Objectives: These post hoc analyses evaluated improvements over time in HRQoL relative to an age/gender-matched normative population in RA pts with inadequate responses to tumor necrosis factor inhibitors (TNF-IR) from the phase 3 SIRROUND-T trial.

Methods: 878 pts received SIR 50mg every 4 weeks (q4w), SIR 100mg every 2 weeks (q2w), or placebo (pbo) q2w. Health-related physical/emotional wellbeing were measured at baseline (BL) and Wk 24 by the 36-item Short Form Questionnaire (SF-36), fatigue by Functional Assessment of Chronic Illness Therapy (FACIT)-Fatigue (FACIT-F), and physical function by Health Assessment Questionnaire-Disability Index (HAQ-DI).

Results: SF-36 physical and mental component summary (PCS and MCS) mean scores at BL for pbo, SIR $50 \mathrm{mg} \mathrm{q} 4 \mathrm{w}$, and $100 \mathrm{mg}$ q2w indicated substantial impairment (PCS: 33.2, 31.8, and 32.4; MCS: 41.9, 41.2, and 42.1). Significantly greater improvements from BL were reported at Wk 24 with SIR $50 \mathrm{mg} \mathrm{q} 4 \mathrm{w}$ and $100 \mathrm{mg}$ q $2 \mathrm{w}$ vs pbo in PCS (4.8 and 5.1 vs 1.7$)$ and MCS (3.9 and 4.0 vs 1.1) mean scores (all $P<0.001$ ), exceeding the minimum clinically important difference (MCID) of 2.5. Significantly greater least squares mean changes in the 8 SF-36 domain raw scores were reported with both doses of SIR vs pbo at Wk 24; all were $>$ MCID of 5.0 (Table; Figure). More pts receiving SIR $50 \mathrm{mg} \mathrm{q4w}$ or $100 \mathrm{mg}$ q2w reported SF-36 domain scores $\geq$ normative values (ranges: $11-34 \%$ and $13-42 \%$ ) vs pbo (range: 6-29\%). For pbo, SIR 50mg q4w, and SIR 100mg q2w, BL FACIT-F scores were $26.0,24.2$, and 25.2 ; clinically meaningful improvements $>$ MCID (4 points) were reported by 54.3 and $51.4 \%$ of pts receiving SIR $50 \mathrm{mg} \mathrm{q} 4 \mathrm{w}$ and $100 \mathrm{mg}$ q2w vs $33.7 \%$ with pbo $(P<0.001)$. Numerically greater percentages of pts reported scores $\geq$ normative values with both doses of SIR vs pbo (27 and $28 \%$ vs $16 \%$ ). BL HAQ-DI scores were $1.57,1.65$, and 1.61 with pbo, SIR $50 \mathrm{mg}$ $\mathrm{q} 4 \mathrm{w}$, and $100 \mathrm{mg} \mathrm{q} 2 \mathrm{w}$. Clinically meaningful improvements (change of $\leq-0.22$ ) were reported by significantly higher proportions of pts receiving SIR $50 \mathrm{mg} q 4 \mathrm{w}$ $(52.2 \%)$ or $100 \mathrm{mg} \mathrm{q2w}(54.8 \%)$ vs pbo $(37.4 \% ; P<0.001)$. Numerically more pts reported HAQ-DI scores $\geq$ normative values with SIR $50 \mathrm{mg} \mathrm{q4W}$ and $100 \mathrm{mg}$ q2W vs pbo ( 13 and $16 \%$ vs $9 \%$ ).

Table 1. Improvements in SF-36 Domain Scores at Wk 24 (all $P<0.001$ )

\begin{tabular}{lccc}
\hline Domain & LSM change SIR 50mg q4w & LSM change SIR $100 \mathrm{mg}$ q2w & LSM change pbo \\
\hline Physical function & 9.38 & 10.75 & 0.47 \\
Role-physical & 12.85 & 13.52 & 5.03 \\
Bodily pain & 17.66 & 17.51 & 7.46 \\
General health & 6.81 & 7.76 & 1.57 \\
Vitality & 10.10 & 9.68 & 4.14 \\
Social function & 12.40 & 11.75 & 3.68 \\
Role-emotional & 9.29 & 9.86 & 0.42 \\
Mental health & 6.73 & 7.96 & 2.10 \\
\hline
\end{tabular}

LSM, least squares mean.

Figure. SF-36 domains at Wk 24 for SIR 50mg $q 4 w$ and SIR $100 \mathrm{mg} q 2 w$

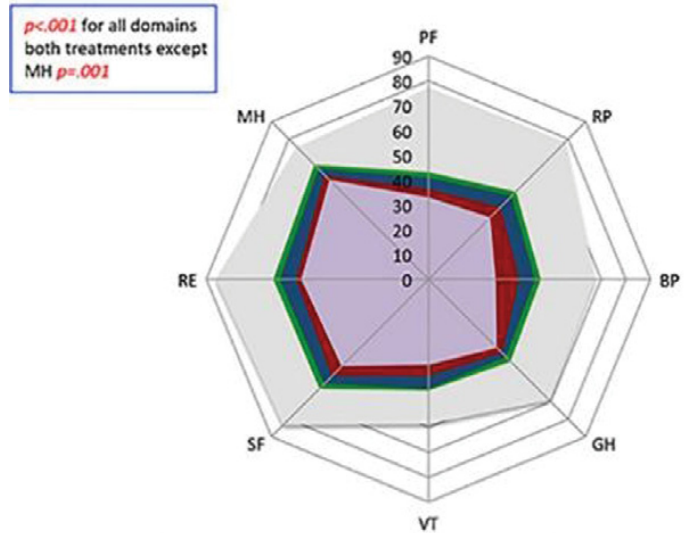

AVG Norms 3003 = S1R $100 \mathrm{mg}$ q 2W -WK24 a S1R S0mg q4W -WK24 = Placebo - WK24 = Combined Baseline

Conclusions: In TNF-IR RA pts, SIR treatment resulted in greater and clinically meaningful improvements in HRQoL vs pbo that met or exceeded population normative values, with similar results for SIR $50 \mathrm{mg} \mathrm{q} 4 \mathrm{w}$ and $100 \mathrm{mg}$ q2w. Disclosure of Interest: V. Strand Consultant for: Abbvie, Amgen, AstraZeneca, Biogenldec, Boehringer Ingelheim, Celltrion, Crescendo, Genentech/Roche, GSK, 